\title{
The Predictive Value of MLR for Radiation Pneumonia During Radiotherapy of Thoracic Tumor Patients
}

This article was published in the following Dove Press journal: Cancer Management and Research

Ya Gao
Xinyi Wu
Yunhao Li
Yifei Li
Qingyu Zhou
Qiongqiong Wang
Chaoyi Wei
Deli Shi
Congying Xie
Huanle Pan
Department of Radiotherapy and
Chemotherapy, The First Affiliated
Hospital of Wenzhou Medical University,
Wenzhou, Zhejiang, People's Republic of
China

Correspondence: Congying Xie; Huanle Pan

Department of Radiotherapy and Chemotherapy, The First Affiliated Hospital of Wenzhou Medical University, No. 2 Fuxue Street, Wenzhou, Zhejiang 325000, People's Republic of China Tel +86 I38 677I I88I; +86I5088980299 Fax +86 57788069372

Email wzxiecongying@163.com; phlphh@I63.com
Purpose: To evaluate the predictive value of blood lymphocyte, monocyte to lymphocyte ratio (MLR), and neutrophil to lymphocyte ratio (NLR) for radiation pneumonia (RP) in patients with thoracic tumors receiving radiotherapy.

Patients and Methods: The clinical data of 65 patients with thoracic tumor (esophageal cancer, lung cancer) treated by radiotherapy in our hospital were retrospectively analyzed. Patients were divided into the RP group and the non-RP group according to the Common Terminology Criteria for Adverse Events (CTCAE 5.0). Data on blood cell counts, including lymphocytes, monocytes, and neutrophils, were collected before ( 0 weeks) and after 1, 2, and 4 weeks of radiotherapy.

Results: Of the 65 patients enrolled, 27 developed radiation pneumonia and 38 did not. Patients' clinical factors, including age, TNM stage, tumor type, underlying lung disease, and history of smoking, had no correlation with RP. ANOVA of repeated measurement data showed that the changes of MLR in the group with RP during radiotherapy were significantly different from those in the non-RP group $(P<0.05)$. The RP prediction model based on the identified risk factors was established using receiver operator characteristic curves. The results showed that the area under the curve for the monocyte to lymphocyte ratio was 0.755 (95\% CI, 0.63-0.87, $P=0.000)$, and the best cutoff point for MLR was 0.426 .

Conclusion: MLR could predict radiation pneumonia in patients with thoracic tumor radiotherapy and achieve early monitoring, early prevention, and treatment.

Keywords: cancer, radiotherapy, radiation pneumonitis, monocyte to lymphocyte ratio

\section{Introduction}

Radiotherapy (RT) is a major therapeutic strategy for patients with lung cancer. Radiation-induced lung injury (RILI) is a common dose-limiting complication of thoracic radiotherapy that usually consists of radiation pneumonitis (RP) and radiation fibrosis (RF). ${ }^{1-4}$ The symptoms of RP are cough, asthma, and even respiratory failure. It affects quality of life greatly, and may even lead to death. ${ }^{5}$ Recent clinical data have demonstrated that the incidence of irradiation-related pulmonary injury among patients with cancer who received radiotherapy ranged from $20.3 \%$ to $36.9 \%{ }^{6-8}$

More and more studies suggest that the inflammation background of the host had an influence on the incidence of RILI. ${ }^{9,10}$ Some evidence from preclinical and clinical investigations supports that recruitment or local infiltration of $\mathrm{T}$ lymphocytes had a causal connection with the complex inflammation course of 
$\mathrm{RP}$ and became a common characteristic of RP. ${ }^{11}$ More importantly, detection of $\mathrm{T}$ lymphocyte subsets undertaken by flow cytometric analysis found that $\mathrm{CD}^{+}$ $\mathrm{T}$ lymphocytes remarkably accumulated during the radioactive pneumonia period in patients with lung cancer. ${ }^{12}$ Zhou et al's research found that lower peripheral blood levels of lymphocytes and $\mathrm{CD}^{+} \mathrm{T}$ lymphocytes were associated with an increased risk of RP, which was associated with differences in radiation-induced lung toxicity among individuals and helped identify those who were susceptible to developing RP after RT. ${ }^{13}$ In addition, CBCderived inflammatory biomarkers such as the systemic immune inflammation index (SII), neutrophil to lymphocyte ratio (NLR), platelet to lymphocyte ratio (PLR), and monocyte to lymphocyte ratio (MLR) were also used as prognostic factors in various malignancies. ${ }^{14-16}$ These biomarkers are based on two or three parameters related to neutrophils, lymphocytes, platelets, and monocytes. NLR, PLR, and MLR have been used as markers in systemic inflammation and are associated with poor outcomes in solid malignancies. ${ }^{14}$ The neutrophil to lymphocyte ratio (NLR), which involves measurement of a subgroup of white blood cells, has been identified as a marker of systemic inflammation. Lee et al conducted a retrospective analysis of patients with stage III nonsmall cell lung cancer receiving concurrent chemoradiation and found that patients with symptomatic radiation pneumonitis had higher NLR and C-reactive protein levels ( $P=0.067$ and $P=0.012$, respectively). ${ }^{17}$ The NLR was statistically significant for progression of radiation pneumonia to symptomatic pneumonia. Pan et al found that combining NLR, V20, and mean lung dose could predict radiation-induced lung injury in lung cancer patients receiving concurrent IMRT and chemotherapy. ${ }^{18}$ It was also found that the pre-treatment NLR level was a representative indicator of systemic inflammation and was associated with RILI grade 3 or higher. However, the relationship between MLR and RILI has not been well discussed before.

We retrospectively analyzed changes of serum levels of lymphocyte, NLR, and MLR during the radiotherapy treatment period in 65 patients enrolled in the present study. Meanwhile, the prediction model based on the identified risk factors was established using receiver operator characteristic curves. We intended to explore an economic and convenient prediction model of radiation pneumonitis during radiotherapy (RT) in thoracic malignant tumor patients.

\section{Patients and Methods}

\section{Patient Population}

Sixty-five pathologically confirmed patients with esophageal and lung cancer treated in the First Affiliated Hospital of Wenzhou Medical University from 2014 to 2019 were retrospectively collected. The inclusion conditions were as follows: patients with thoracic malignancies receiving radiation therapy who had an expected survival of more than 6 months and an ECOG score of $\leq 4$. We excluded those who had incomplete RT, inflammation, or who had hematological diseases. All patients signed informed consent. The study was approved by the Ethics Committee of the First Affiliated Hospital of Wenzhou Medical University.

\section{Treatment Regimen}

All patients received Intensity Modulated Radiation Therapy (IMRT) and 3-dimensional conformal radiation therapy (3D-CRT). The total tumor volume (GTV), clinical volume (CTV), and planned target volume (PTV) were determined according to the definitions reported in ICRU 50 and 62. The median dose of radiotherapy was 56 Gy. The criteria for receiving a treatment plan are: at least $99 \%$ of the planned target volume (PTV) is covered by $95 \%$ of the prescribed dose, and the maximum dose is less than or equal to $105 \%$. We apply normal tissue dosevolume restrictions. The limited volume of the spinal cord was not allowed to be more than $45 \mathrm{~Gy}$. The average dose to the heart receiving more than 35 Gy was limited. The average esophagus dose should not exceed 34 Gy. Normal lung total volume was defined as the volume of both lungs minus the total target volume (GTV). V20 was no more than $30 \%$ in both lungs, V5 $\leq 50 \%$, and the average dose was no more than $17 \mathrm{~Gy}$. The dosage of RT was delivered with $6 \mathrm{MV}$ X-ray by linear accelerator.

\section{Detection of Peripheral Blood}

Data on blood cell counts of all patients were performed in peripheral blood samples using standard phlebotomy techniques at four time points: at baseline (within 1 week prior to irradiation), and at an interval of 1, 2, and 4 weeks during RT. The blood lymphocyte, mononuclear cell, and neutrophil subsets of 65 patients were detected at the same time points.

\section{Diagnosis of Radiation-Induced Lung Injury}

History, physical examination, and chest CT scan were checked at each follow-up. Generally, chest CT scans 
were performed 1, 3, and 6 months after radiotherapy. In acute pneumonia, chest CT showed hyperemia and exudation of the lung, increased texture and small pieces of shadow, and the edge was fuzzy. If the treatment continued without remission, chronic pulmonary fibrosis would appear, which was characterized by a strip shadow in the radiation area, fiber contraction, mediastinal or tracheal displacement, and grid or honeycomb changes in severe cases. Generally, 1-2 grade radiation lung injury had no clinical symptoms and did not require routine clinical treatment. However, patients with grade 3 and above had obvious dry cough, shortness of breath, and symptoms of hypoxia, requiring clinical treatment. If the patient developed fever, dry cough, and other suspected symptoms during radiotherapy, chest CT should be used to observe lung images, and blood routine was also performed. If CRP and PCT were increased, it often indicated bacterial infection, and further identification of sputum culture. RP was diagnosed by CTCAE 5.0 evaluation criteria as follows: continuous cough and/or dyspnea occur in patients with radiation pneumonia of grade $(\geq 2)$ and need to be treated clinically. The chest CT scan showed a patchy or strip shadow in the radiation area, and honeycomb-like changes in severe cases.

\section{Statistical Analysis}

SPSS 25.0 and GraphPad Prism 8.0.2 were adopted to analyze the data. The chi-square test was performed to test the categorical variables. Repeated measures of analysis of variance (ANOVA) was performed to detect whether serum indicators had statistical significance between the two groups. The ROC curve prediction model was established, and the predictive value of serum inflammatory maker on radiation pneumonitis was evaluated by analyzing the area under the curve. All $P$ values less than 0.05 were considered statistically significant.

\section{Results}

A total of 65 patients with lung cancer and esophageal cancer were enrolled, of which 39 were lung cancer and 26 were esophageal cancer. The characteristics of the patients are shown in Table 1 . There were 59 male patients and 6 female patients, with a median age of 65 years (ranging from 43 to 86 years). Of these 65 patients, 55 received concurrent chemoradiotherapy and 10 received radiotherapy alone. A total $41.5 \%$ of these patients received 50 to $60 \mathrm{~Gy}$ of radiation, and $35.3 \%$ received no less than $60 \mathrm{~Gy}$. The median radiotherapy dose was 56 Gy. According to the general term standard for adverse events (CTCAE, version 5.0), patients with radiation pneumonia were evaluated. In total, 27 patients $(41.5 \%)$ developed grade 2 or higher RP, including 17 cases of grade 2, 5 cases of grade 3,3 cases of grade 4, and 2 cases of grade 5. These events were frequently observed within 1 to 3 months after radiotherapy. There was no significant difference in gender, age, tumor type, TNM stage, and treatment mode between the two groups, while the ECOG score was significantly different between the two groups $(P<0.05)$.

\section{Changes in Lymphocyte Levels During Radiotherapy}

Figure 1 presents the level of lymphocyte in patients during radiotherapy between the two groups. Our study illustrated that there was no significant difference in lymphocytes between the RP and non-RP groups. Serum lymphocyte concentrations in both groups were lower than $2.0 \times 10^{9} / \mathrm{L}$.

\section{Changes in NLR Levels During Radiotherapy}

The NLR value of the RP group decreased to a low value within the first week of radiotherapy, with an inflection point, and then showed a continuous trend of increasing (Figure 2). While in patients with non-RP, the NLR value showed a continuous and slowly increasing trend, and no obvious inflection point appeared. The NLR final value of patients with RP after 4 weeks of radiotherapy was higher than that of the non-RP group. However, the difference of NLR between the two groups was not significant $(P>0.05)$.

\section{Changes in MLR Levels During Radiotherapy}

Different from the level of lymphocyte and NLR, there was a statistically significant difference in the level of MLR between the RP group and the non-RP group after 2 weeks of radiotherapy $(P<0.05)$. Results demonstrated that the final MLR value of patients with RP was higher, and the difference was statistically significant $(P<0.05)$, which is shown in Figure 3.

\section{Predictive Risk Assessment Model of RP}

In order to establish a better prediction model, we established a ROC curve for MLR. The results showed that the area under the curve for the monocyte to lymphocyte ratio was $0.755(95 \% \mathrm{CI}, 0.63-0.87, P=0.000)$, and the best 
Table I Patients Characteristics $(n=65)$

\begin{tabular}{|c|c|c|c|c|c|}
\hline Characteristics & $R P(N=27)$ & Non-RP $(\mathrm{N}=38)$ & Total $(\mathrm{N}=65)$ & $\chi^{2}$ & $P$ values \\
\hline $\begin{array}{l}\text { Gender } \\
\text { Male } \\
\text { Female }\end{array}$ & $\begin{array}{l}26 \\
1\end{array}$ & $\begin{array}{l}33 \\
5\end{array}$ & $\begin{array}{l}59 \\
6\end{array}$ & 0.745 & 0.388 \\
\hline $\begin{array}{l}\text { Age, years } \\
<65 \\
\geq 65\end{array}$ & $\begin{array}{l}13 \\
14\end{array}$ & $\begin{array}{l}17 \\
21\end{array}$ & $\begin{array}{l}30 \\
35\end{array}$ & 0.074 & 0.786 \\
\hline $\begin{array}{r}\text { ECOG } \\
0-2 \\
3-4\end{array}$ & $\begin{array}{l}17 \\
10\end{array}$ & $\begin{array}{l}34 \\
4\end{array}$ & $\begin{array}{l}51 \\
14\end{array}$ & 6.565 & $0.01 *$ \\
\hline $\begin{array}{l}\text { Smoke } \\
\text { Yes } \\
\text { No }\end{array}$ & $\begin{array}{l}14 \\
13\end{array}$ & $\begin{array}{l}24 \\
14\end{array}$ & $\begin{array}{l}38 \\
27\end{array}$ & 0.831 & 0.362 \\
\hline $\begin{array}{l}\text { Chemotherapy } \\
\text { Yes } \\
\text { No }\end{array}$ & $\begin{array}{l}22 \\
5\end{array}$ & $\begin{array}{l}33 \\
5\end{array}$ & $\begin{array}{l}55 \\
10\end{array}$ & 0.058 & 0.809 \\
\hline $\begin{array}{l}\text { Basic lung disease } \\
\text { Yes } \\
\text { No }\end{array}$ & $\begin{array}{l}2 \\
25\end{array}$ & $\begin{array}{l}2 \\
36\end{array}$ & $\begin{array}{l}4 \\
61\end{array}$ & 0.000 & 1.000 \\
\hline $\begin{array}{l}\text { TNM } \\
\text { I-II } \\
\text { III-IV }\end{array}$ & $\begin{array}{l}8 \\
19\end{array}$ & $\begin{array}{l}7 \\
31\end{array}$ & $\begin{array}{l}15 \\
50\end{array}$ & 1.117 & 0.291 \\
\hline $\begin{array}{l}\text { GTV dose (Gy) } \\
\quad<56 \\
\geq 56\end{array}$ & $\begin{array}{l}14 \\
13\end{array}$ & $\begin{array}{l}16 \\
22\end{array}$ & $\begin{array}{l}30 \\
35\end{array}$ & 0.698 & 0.403 \\
\hline $\begin{array}{l}\text { Tumor type } \\
\text { Esophageal } \\
\text { Lung cancer }\end{array}$ & $\begin{array}{l}12 \\
15\end{array}$ & $\begin{array}{l}14 \\
24\end{array}$ & $\begin{array}{l}26 \\
39\end{array}$ & 0.380 & 0.538 \\
\hline
\end{tabular}

Note: $* P<0.05$.

Abbreviations: RP, radiation pneumonia; GTV, gross tumor volume.

cutoff point for MLR was 0.426 . The ROC curve is shown in Figure 4.

\section{Discussion}

The mechanism of radiation-induced lung injury is still unclear, but it is currently recognized that its occurrence was a complex process involving a variety of cytokines and immune and inflammatory factors, especially inflammatory cytokines and lymphocytes, which caused damage to endothelial cells, parenchymal cells, vasculature, stroma, and the tissue microenvironment. ${ }^{19}$ In view of the pathological development of the disease, patients with advanced pulmonary fibrosis have a poor quality of life and a high mortality rate. In view of the above facts, there is an urgent need to explore prognostic biomarkers that are easily evaluated for patients with radiotherapy.

In this study, changes in MLR during radiotherapy were statistically different in the group with and without RP. Especially 2 weeks after radiotherapy, the difference between the two groups was obvious. MLR values started to increase in both groups after radiotherapy, but the patients in the RP group had a relatively slow upward trend at 1 week after radiotherapy, and then increased rapidly, and the final value was higher than that in the nonRP group. However, the difference was not statistically significant in lymphocytes. We found that after the start of radiotherapy, lymphocytes in both groups showed a decreasing trend. The decline in the RP group was relatively gentle and the final value was lower at 4 weeks. Zhou et al's clinical retrospective study found 


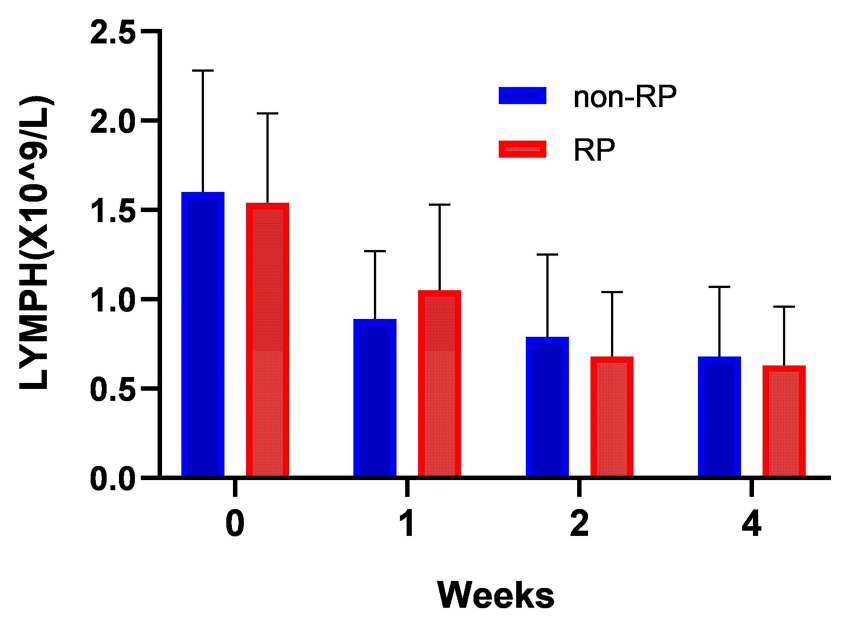

Figure I Changes in lymphocyte levels during radiotherapy. There is no statistically significant difference in the level of lymphocyte between the RP group and the nonRP group $(P>0.05)$.

Abbreviations: RP, radiation pneumonia; LYMPH, lymphocyte absolute value.

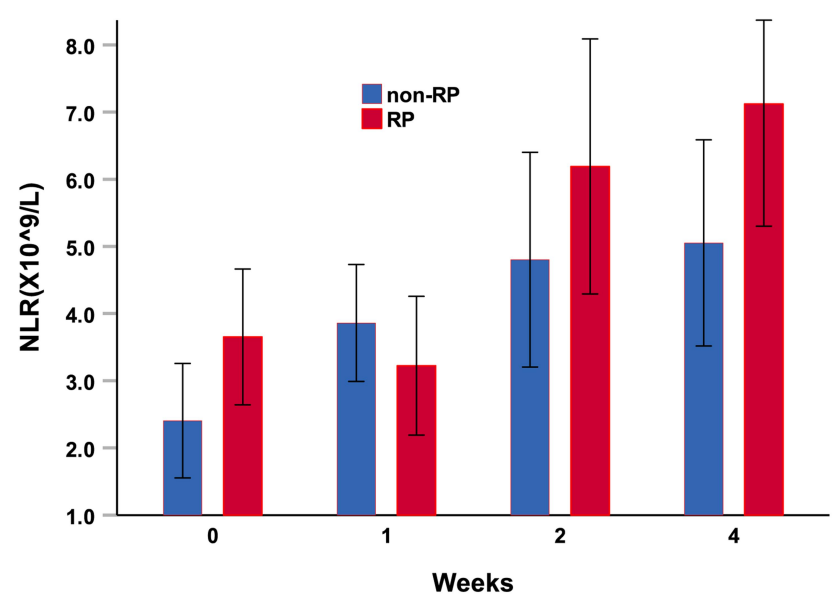

Figure 2 Changes in NLR levels during radiotherapy. The serum level of NLR between the RP group and the non-RP group $(P>0.05)$.

Abbreviations: RP, radiation pneumonia; NLR, neutrophil to lymphocyte ratio.

that lymphocyte levels decreased markedly in patients who developed grade 2 or 3 RP compared with patients with grade 0 or $1 \mathrm{RP}$ from the second to the sixth week of the RT start. ${ }^{13}$ Furthermore, they found a sharp increase in WBCs and neutrophils at week 12 post irradiation in patients who experienced grade 3 RP. Maybe it was due to the lack of time nodes in our study, which was inconsistent with previous research. Numerous research studies showed that a decrease in circulating lymphocytes could be due to lymphocytes infiltrating into the pulmonary interstitium, and it may reasonably explain the decrease in circulating lymphocytes during pre-RP and RP..$^{20,21}$ Radiation activates the inflammatory cascade, neutrophils can secrete cytokines, and chemokines mediate the

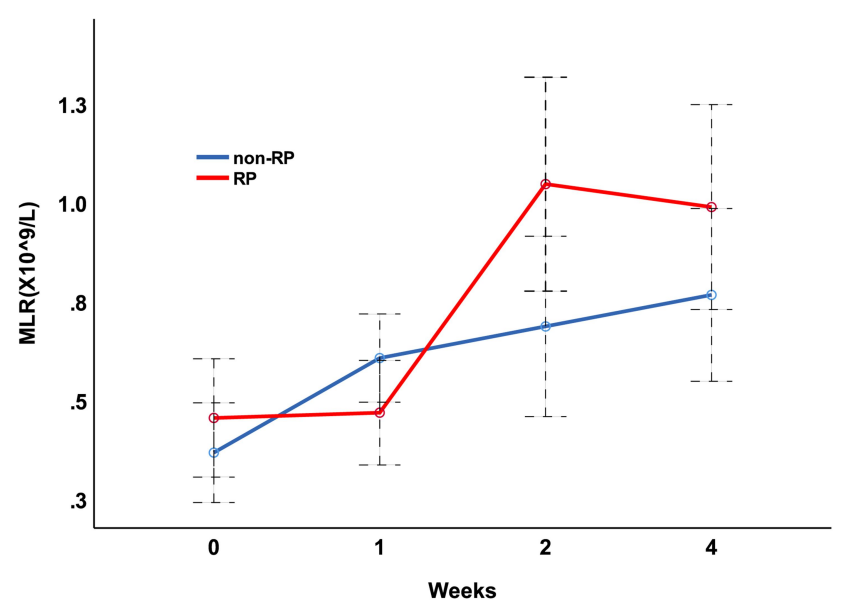

Figure 3 Changes in MLR levels during radiotherapy. The serum level of MLR between the RP group and the non-RP group $(P<0.05)$.

Abbreviations: RP, radiation pneumonia; MLR, monocyte to lymphocyte ratio.

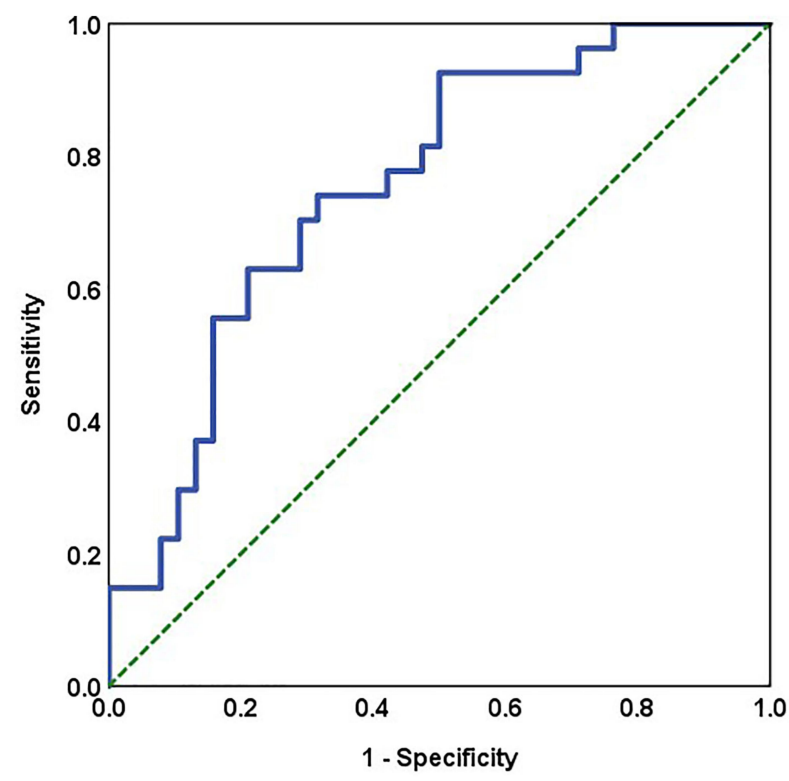

Figure 4 Predictive risk assessment model. ROC curve based on the sensitivity and specificity of MLR to predict RP.

Abbreviations: ROC curve, receiver operating characteristic curve; MLR, monocyte to lymphocyte ratio.

recruitment of inflammatory cells and angiogenesis. In addition, elevated neutrophils can suppress the cytolytic activity of lymphocytes, natural killer cells, activated $\mathrm{T}$ cells, and suppression of adaptive immune responses. ${ }^{22,23}$ Current studies have shown that NLR can predict the occurrence of radiation pneumonia in patients with tumor radiotherapy. ${ }^{17,18}$ Lee et al pointed out that NLR combined with dosimetric factors could predict symptomatic radiation pneumonitis in patients with NSCLC who received concurrent radiotherapy and chemotherapy. ${ }^{17}$ Pan et al found that combining NLR, 
V20, and mean lung dose could predict radiation-induced lung injury in lung cancer patients receiving concurrent IMRT and chemotherapy. ${ }^{18}$ Multivariate analysis showed that total dose $\geq 60$ Gy, V20 $\geq 20 \%$, mean lung dose $\geq 12$ Gy, and NLR $\geq 2.2$ were still independent predictors of RP $(P=0.010,0.043,0.028$, and 0.015 , respectively). It was also found that the pre-treatment NLR level was a representative indicator of systemic inflammation and was associated with RILI grade 3 or higher. However, the exact mechanism of this effect remains unclear. The most likely reason is that the host's inflammatory response is involved in the occurrence and development of RP.

In our study, it was also found that the baseline NLR and MLR levels of patients with RP before radiotherapy (week 0) were higher than those without RP, and the baseline NLR level was greater than 2.2; however, further research was still needed to confirm. In order to improve the predictive power of RP, analysis of MLR was carried out by ROC model. The area under the combined curve of MLR was 0.755 (95\% CI, 0.63-0.87, $P=0.000$ ). The results show that MLR could further improve the prediction ability. In addition, we have found the ECOG in the clinical data was significantly different between the two groups $(P<0.05)$. Deng et al found that only COPD had a significant difference between the two groups with and without radiation pneumonia, and no significant abnormalities were found in smoking and chemotherapy. ${ }^{24}$ Otherwise, few studies currently suggest that certain chemotherapy and concurrent chemotherapy may increase the risk of RP. ${ }^{25}$ Some limitations existed in the present study. First, the number of patients was small. Second, this study was limited by selection biases due to its single-center and retrospective nature. Thus, prospective studies to verify the impact of MLR level in thoracic tumor patients treated by radiotherapy are needed to confirm these preliminary results.

\section{Conclusion}

In conclusion, this study indicates that MLR may be an available serum biomarker in the early stage of RT, which may provide a convenient and non-invasive method for early detection, early intervention, and treatment in patients who undergo thoracic radiotherapy.

\section{Funding}

The study is partially supported by funding from Wenzhou Municipal Science and Technology Bureau (H20180003).

\section{Disclosure}

The authors report no conflicts of interest in this work.

\section{References}

1. Puthawala K, Hadjiangelis N, Jacoby SC, et al. Inhibition of integrin alpha(v)beta6, an activator of latent transforming growth factor-beta, prevents radiation-induced lung fibrosis. Am J Respir Crit Care Med. 2008;177(1):82-90. doi:10.1164/rccm.200706-806OC

2. Christofidou-Solomidou M, Tyagi S, Tan KS, et al. Dietary flaxseed administered post thoracic radiation treatment improves survival and mitigates radiation-induced pneumonopathy in mice. BMC Cancer. 2011;11:269. doi:10.1186/1471-2407-11-269

3. Mathew B, Jacobson JR, Berdyshev E, et al. Role of sphingolipids in murine radiation-induced lung injury: protection by sphingosine 1-phosphate analogs. FASEB J. 2011;25(10):3388-3400. doi:10.10 96/fj.11-183970

4. Oh JH, Craft JM, Townsend R, et al. A bioinformatics approach for biomarker identification in radiation-induced lung inflammation from limited proteomics data. J Proteome Res. 2011;10:1406-1415. doi:10.1021/pr101226q

5. Shi A, Zhu G, Wu H, et al. Analysis of clinical and dosimetric factors associated with severe acute radiation pneumonitis in patients with locally advanced non-small cell lung cancer treated with concurrent chemotherapy and intensity-modulated radiotherapy. Radiat Oncol. 2010;5(1):35. doi:10.1186/1748-717X-5-35

6. Matsuo Y, Shibuya K, Nakamura M, et al. Dose-volume metrics associated with radiation pneumonitis after stereotactic body radiation therapy for lung cancer. Int J Radiat Oncol Biol Phys. 2012;83: e545-e549. doi:10.1016/j.jjrobp.2012.01.018

7. Minor GI, Yashar CM, Spanos WJ Jr, et al. The relationship of radiation pneumonitis to treated lung volume in breast conservation therapy. Breast J. 2006;12:48-52. doi:10.1111/j.1075-122X.2006. 00180.x

8. Rosenzweig KE, Zauderer MG, Laser B, et al. Pleural intensity-modulated radiotherapy for malignant pleural mesothelioma. Int J Radiat Oncol Biol Phys. 2012;83:1278-1283. doi:10.1016/j.ijrobp.2011.09.027

9. Hill RP, Zaidi A, Mahmood J, Jelveh S. Investigations into the role of inflammation in normal tissue response to irradiation. Radiother Oncol. 2011;101:73-79. doi:10.1016/j.radonc.2011.06.017

10. Abernathy LM, Fountain MD, Rothstein SE, et al. Soy isoflavones promote radioprotection of normal lung tissue by inhibition of radiation-induced activation of macrophages and neutrophils. $J$ Thorac Oncol. 2015;10:1703-1712. doi:10.1097/JTO.000000000 0000677

11. Ozsahin M, Crompton NE, Gourgou S, et al. CD4 and CD8 T-lymphocyte apoptosis can predict radiation-induced late toxicity: a prospective study in 399 patients. Clin Cancer Res. 2005;11:7426-7433. doi:10.1158/1078-0432.CCR-04-2634

12. Gibson PG, Bryant DH, Morgan GW, et al. Radiation-induced lung injury: a hypersensitivity pneumonitis? Ann Int Med. 1988;109: 288-291. doi:10.7326/0003-4819-109-4-288

13. Zhou P, Chen L, Yan D, et al. Early variations in lymphocytes and $\mathrm{T}$ lymphocyte subsets are associated with radiation pneumonitis in lung cancer patients and experimental mice received thoracic irradiation. Cancer Med. 2020;9(10):3437-3444. doi:10.1002/cam4.2987

14. Dolan RD, Lim J, McSorley ST, et al. The role of the systemic inflammatory response in predicting outcomes in patients with operable cancer: systematic review and meta-analysis. Sci Rep. 2017;7:16717.

15. Yuan C, Li N, Mao X, et al. Elevated pretreatment neutrophil/white blood cell ratio and monocyte/lymphocyte ratio predict poor survival in patients with curatively resected non-small cell lung cancer: results from a large cohort. Thorac Cancer. 2017;8(4):350-358. doi:10.1111/ $1759-7714.12454$ 
16. Zhang Y, Chen B, Wang L, et al. Systemic immune-inflammation index is a promising noninvasive marker to predict survival of lung cancer: a meta-analysis. Medicine. 2019;98(3):e13788. doi:10.1097/ MD.0000000000013788

17. Lee YH, Choi HS, Jeong H, et al. Neutrophil-lymphocyte ratio and a dosimetric factor for predicting symptomatic radiation pneumonitis in non-small-cell lung cancer patients treated with concurrent chemoradiotherapy. Clin Respir J. 2018;12:1264-1273. doi:10.1111/ crj. 12660

18. Pan W-Y, Bian C, Zou G-L, et al. Combing NLR, V20 and mean lung dose to predict radiation induced lung injury in patients with lung cancer treated with intensity modulated radiation therapy and chemotherapy. Oncotarget. 2017;8:81387-81393. doi:10.18632/ oncotarget.19032

19. Bai L, Zhou B-S, Zhao Y-X, et al. Dynamic changes in T-cell subsets and C-reactive protein after radiation therapy in lung cancer patients and correlation with symptomatic radiation pneumonitis treated with steroid therapy. Cancer Manag Res. 2019;11:7925-7931. doi:10.2147/CMAR.S209286

20. Martin C, Romero S, Sanchez-Paya J, Massuti B, Arriero JM, Hernandez L. Bilateral lymphocytic alveolitis: a common reaction after unilateral thoracic irradiation. Eur Respir J. 1999;13:727-732. doi:10.1034/j.1399-3003.1999.13d05.x
21. Morgan GW, Breit SN. Radiation and the lung: a reevaluation of the mechanisms mediating pulmonary injury. Int $J$ Radiat Oncol Biol Phys. 1995;31:361-369. doi:10.1016/0360-3016(94)00477-3

22. Mishalian I, Granot Z, Fridlender ZG. The diversity of circulating neutrophils in cancer. Immunobiology. 2017;222:82-88. doi:10.1016/ j.imbio.2016.02.001

23. Grommes J, Soehnlein O. Contribution of neutrophils to acute lung injury. Mol Med. 2011;17:293-307.

24. Deng YX, Qiu T, Patel N, et al. Clinical management of risk of radiation pneumonia with serum markers during the radiotherapy for patients with thoracic malignant tumors. Cancer Manag Res. 2019;11:10249-10256. doi:10.2147/CMAR.S231995

25. McCurdy M, McAleer MF, Wei W, et al. Induction and concurrent taxanes enhance both the pulmonary metabolic radiation response and the radiation pneumonitis response in patients with esophagus cancer. Int J Radiat Oncol Biol Phys. 2010;76(3):16-823. doi:10.10 16/j.ijrobp.2009.02.059

\section{Publish your work in this journal}

Cancer Management and Research is an international, peer-reviewed open access journal focusing on cancer research and the optimal use of preventative and integrated treatment interventions to achieve improved outcomes, enhanced survival and quality of life for the cancer patient.
The manuscript management system is completely online and includes a very quick and fair peer-review system, which is all easy to use. Visit http://www.dovepress.com/testimonials.php to read real quotes from published authors. 